\title{
HERMES results on transverse target single-spin asymmetries in inclusive electroproduction of charged pions and kaons
}

\author{
Klaus Rith* (on behalf of the HERMES collaboration) \\ Friedrich-Alexander-Universität Erlangen-Nürnberg \\ E-mail: klaus.rith@physik.uni-erlangen.de
}

\begin{abstract}
Single-spin asymmetries were investigated in inclusive electroproduction of charged pions and kaons from transversely polarised protons at the HERMES experiment. The asymmetries were studied as a function of the azimuthal angle $\Psi$ around the beam direction between the target-spin direction and the hadron production plane, the transverse hadron momentum $P_{T}$ relative to the direction of the incident beam, and the Feynman variable $x_{F}$. The $\sin \Psi$ amplitudes are positive for $\pi^{+}$and $K^{+}$, slightly negative for $\pi^{-}$and consistent with zero for $K^{-}$, with particular $P_{T}$ but weak $x_{F}$ dependences. Large asymmetries are observed for two small subsamples of events, where also the scattered electron was recorded by the spectrometer.
\end{abstract}

XXII. International Workshop on Deep-Inelastic Scattering and Related Subjects, 28 April - 2 May 2014

Warsaw, Poland

\footnotetext{
* Speaker.
} 


\section{Introduction}

Left-right cross-section asymmetries $A_{N}$ for the inclusive production of various hadrons in hadron-nucleon collisions with transversely polarised beams or targets have been studied over the past three decades by numerous experiments for centre-of-mass energies in the range 4.9 - 500 $\mathrm{GeV}$. They were found to be positive for $\pi^{+}, \pi^{0}, \eta, K^{+}, K^{-}$, and anti-protons, negative for $\pi^{-}$and neutrons, and compatible with zero for protons. In all non-zero cases, $A_{N}$ increases in magnitude with increasing $P_{T}$ and $x_{F}$, exceeding $\left|A_{N}\right|=0.4$ for charged pions. Here, $P_{T}$ is the transverse momentum of the produced hadron with respect to the direction of the incident hadron and the Feynman variable $x_{F}$ is defined as the ratio of the longitudinal hadron momentum $P_{L}$ along the beam direction to its maximum possible value. A review of experimental results can be found, e.g., in Refs. [1] and [2], together with a discussion on current theoretical work. The theoretical attempts to explain the experimental results include two approaches. One is based on unintegrated, transverse-momentum dependent distribution and fragmentation functions, in particular the chiralodd transversity distribution of transversely polarised quarks in a transversely polarised nucleon, in conjunction with the chiral-odd Collins fragmentation function [3], or the transverse-momentumdependent time-reversal odd Sivers distribution function [4] of unpolarised quarks with non-zero transverse momenta in a transversely polarised nucleon. The latter has originally been invented to explain $A_{N}$ in hadron-nucleon reactions. The other approach links collinear parton dynamics to higher-twist multiparton correlations [5, 6, 7]. Both approaches succeeded in reproducing the existing measurements of $A_{N}$ to a large extent, and have been shown to be related to and consistent with each other in the kinematic region where they both apply [8]. Recently, however, a sign error was identified [9] that invalidates the good agreement observed earlier.

While $A_{N}$ was studied in detail in hadron-nucleon reactions, no experimental information about this quantitity was available until recently from inclusive electroproduction of hadrons, $l p^{\uparrow} \rightarrow h X$. For electroproduction, the theoretical expectations depend on the relative magnitude of the three relevant scales $Q, P_{T}$ and $\Lambda_{Q C D}$, where $-Q^{2}$ is the squared four-momentum of the virtual photon that mediates the lepton-nucleon scattering process and $\Lambda_{Q C D} \cong 0.3 \mathrm{GeV}$ is the QCD scale parameter. The Sivers and Collins effects become significant when $Q^{2}>P_{T}^{2}$ and $Q^{2} \gg \Lambda_{Q C D}^{2}$ and give a contribution that is not $P_{T}$-suppressed. For large $Q^{2}$, the dominant contribution to the asymmetry should therefore come from the Sivers and Collins mechanisms. The single-spin asymmetries measured in semi-inclusive deep-inelastic scattering were, in fact, the basis for an extraction of the Sivers and transversity distribution functions and the Collins fragmentation function (see e.g., Refs. [10, 11]). The twist-three approach leads to a characteristic power suppression by $1 / P_{T}$ for large $P_{T}$, provided $P_{T}$ is the largest scale in the process.

\section{Experiment}

In this contribution, the results from the first measurements of $A_{N}$ in inclusive electroproduction of charged pions and kaons [12] performed by the HERMES experiment are reported. The data were collected with the HERMES spectrometer [13] at the HERA e-p accelerator facility. The $27.6 \mathrm{GeV}$ lepton (electron or positron) beam of HERA was scattered off a transversely polarised gaseous hydrogen target internal to the lepton ring. The average magnitude of the proton- 
polarisation was $S_{T} \approx 0.71$. The direction of the target-spin vector was reversed between the 'upward' and 'downward' directions at 1-3 minute time intervals to minimize systematic effects. The beam was longitudinally polarised, but a helicity-balanced data sample was used to obtain an effectively unpolarised beam.

Selected events had to contain at least one charged-hadron track, identified as either a pion or a kaon, within the angular acceptance of the spectrometer $( \pm 170 \mathrm{mrad}$ horizontally and $\pm(40-140)$ mrad vertically) independent of whether there was also a scattered lepton in the acceptance or not. The trigger of the experiment was formed, for each detector half, by a coincidence of signals from a scintillation counter in front of the spectrometer magnet and from a scintillator hodoscope and a scintillator pre-shower counter behind the magnet with the requirement of an energy deposit greater than $1.4 \mathrm{GeV}$ in an electromagnetic calorimeter. The trigger was almost $100 \%$ efficient for leptons with energies above threshold. The energy threshold of the calorimeter was low enough to trigger also on events with only charged hadrons and no leptons in its geometrical acceptance. In this case, the trigger efficiency was substantially smaller. Averaged over the hadron multiplicity, the trigger efficiency was about $40-45 \%$ for hadron momenta greater than approximately $7 \mathrm{GeV}$ and decreased smoothly with decreasing $P$ to about $15 \%$ at $P \approx 2 \mathrm{GeV}$. Hadrons were distinguished from leptons by using a transition-radiation detector, the scintillator pre-shower counter, and the electromagnetic calorimeter. This resulted in a tiny lepton contamination in the hadron sample of less than $0.1 \%$. Hadrons within the momentum range $2-15 \mathrm{GeV}$ were further identified using a dual-radiator ring-imaging Cherenkov detector. In total, about $60 \cdot 10^{6}\left(50 \cdot 10^{6}\right)$ tracks of positively (negatively) charged pions and $5.1 \cdot 10^{6}\left(2.8 \cdot 10^{6}\right)$ tracks of positively (negatively) charged kaons were collected. These correspond to about $172 \cdot 10^{6}\left(142 \cdot 10^{6}\right)$ positively (negatively) charged pions and $14.5 \cdot 10^{6}\left(7.3 \cdot 10^{6}\right)$ positively (negatively) charged kaons after trigger-efficiency correction.

The cross section for inclusive electroproduction of hadrons using an unpolarised lepton beam and a transversely polarised target includes a polarisation-averaged and a polarisation-dependent part and is given for each hadron species as

$$
\mathrm{d} \sigma\left(P_{T}, x_{F}, \psi\right)=\mathrm{d} \sigma_{U U}\left(P_{T}, x_{F}\right)\left[1+S_{T} A_{U T}^{\sin \psi}\left(P_{T}, x_{F}\right) \sin \psi\right]
$$

Here, the first subscript $U$ denotes unpolarised beam, the second subscript $U(T)$ an unpolarised (transversely polarised) target. The cross section depends on the hadron variables $x_{F}, P_{T}$ and $\psi$, where $\psi$ is the azimuthal angle around the beam direction between the 'upward' target spin direction and the hadron production plane. Note, that in cases where the scattered electron is recorded and the angle between the virtual photon and the lepton beam is small, this angle $\psi$ is approximately equal to the Sivers-angle $\phi-\phi_{s}$, where $\phi\left(\phi_{s}\right)$ is the azimuthal angle about the virtual-photon direction between the lepton scattering plane and the hadron production plane (the 'upward' target spin direction). For a detector with full $2 \pi$-coverage in $\psi$ and constant efficiency, the amplitude $A_{U T}^{\sin \psi}$ and the left-right asymmetry $A_{N}$ along the direction of the incident beam and with respect to the spin direction are related by $A_{N}=-(2 / \pi) A_{U T}^{\sin \psi}$. The $A_{U T}^{\sin \psi}$ amplitudes were extracted by performing a maximum-likelihood fit to the cross section of Eq. 2.1, i.e., the measured yield distribution for the two target-spin states weighted with the inverse of the trigger efficiencies and luminosity, binned in $P_{T}$ and $x_{F}$, but unbinned in $\psi$. 

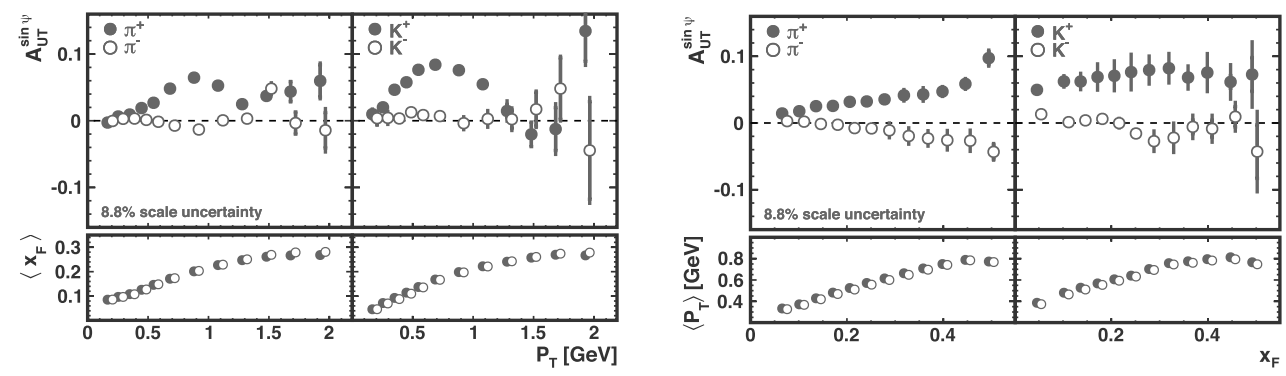

Figure 1: $A_{U T}^{\sin \psi}$ amplitudes for charged pions and kaons as a function of $P_{T}$ (left) and of $x_{F}$ (right). Positive (negative) particles are denoted by closed (open) symbols. The bottom subpanels show the $P_{T}\left(x_{F}\right)$ dependence of the average $x_{F}\left(P_{T}\right)$. Data points for negative particles are slightly shifted horizontally for legibility.

\section{Results}

The extracted $A_{U T}^{\sin \psi}$ amplitudes for charged pions and kaons, integrated over the variable not shown, are presented as a function of $P_{T}\left(x_{F}\right)$ in the left (right) panels of Fig. 1. When visible, the inner error bars show the statistical uncertainties, while the total ones represent the quadratic sum of statistical and systematic uncertainties. The latter include contributions due to corrections for misalignment of the detector, beam position and slope at the interaction point and bending of the beam and the produced hadron in the transverse holding field of the target magnet. Not shown is an additional $8.8 \%$ scale uncertainty due to the precision of the measurement of the target polarisation. The amplitudes are positive for the positive hadrons being slightly larger for kaons compared to pions, while for negative hadrons they are much smaller in magnitude, sometimes positive, sometimes negative. The $P_{T}$ dependences of the amplitudes have a rather peculiar behaviour. The amplitudes vanish for $P_{T} \rightarrow 0$, as they have to by definition. For positive hadrons they increase smoothly with $P_{T}$ up to a maximum value of $0.06(0.08)$ for pions (kaons) at $P_{T} \simeq 0.8 \mathrm{GeV}$ and then decrease again for larger $P_{T}$. For $\pi^{+}$there is an indication of another increase at $P_{T}>1.3 \mathrm{GeV}$. The asymmetries for $\mathrm{K}^{+}$and $\mathrm{K}^{-}$are very different from each other in contrast to the results in pp scattering, where they are rather similar [15]. In the right panels of Fig. 1 the amplitudes are shown as a function of $x_{F}$. For $\pi^{+}\left(\pi^{-}\right)$they increase (decrease) smoothly with $x_{F}$ up to values of about $0.1(-0.04)$. For $K^{+}$, the amplitude is nearly constant around 0.07 while for $K^{-}$it is compatible with zero over most of the $x_{F}$ range.

The variables $x_{F}$ and $P_{T}$ are strongly correlated through the HERMES acceptance as can be seen in the bottom panels of Fig. 1. To separate the kinematical dependences, a two-dimensional extraction of the asymmetries was performed binning simultaneously in $x_{F}$ and $P_{T}$. The results for the extracted asymmetry amplitudes are shown in the left panels of Fig. 2 as a function of $P_{T}$ for four slices in $x_{F}$. For $\pi^{+}$and $K^{+}$, the $P_{T}$ dependence is very similar in all four $x_{F}$ bins, as well in shape as in magnitude, apart from increased statistical fluctuations. Therefore, it can be concluded that the apparent increase of the magnitude of the asymmetry amplitude seen for $\pi^{+}$in the right panel of Fig. 1 is just a reflection of the underlying dependence on $P_{T}$. In contrast, for $\pi^{-}$the decrease with $x_{F}$ follows the one observed in the one-dimensional extraction. 

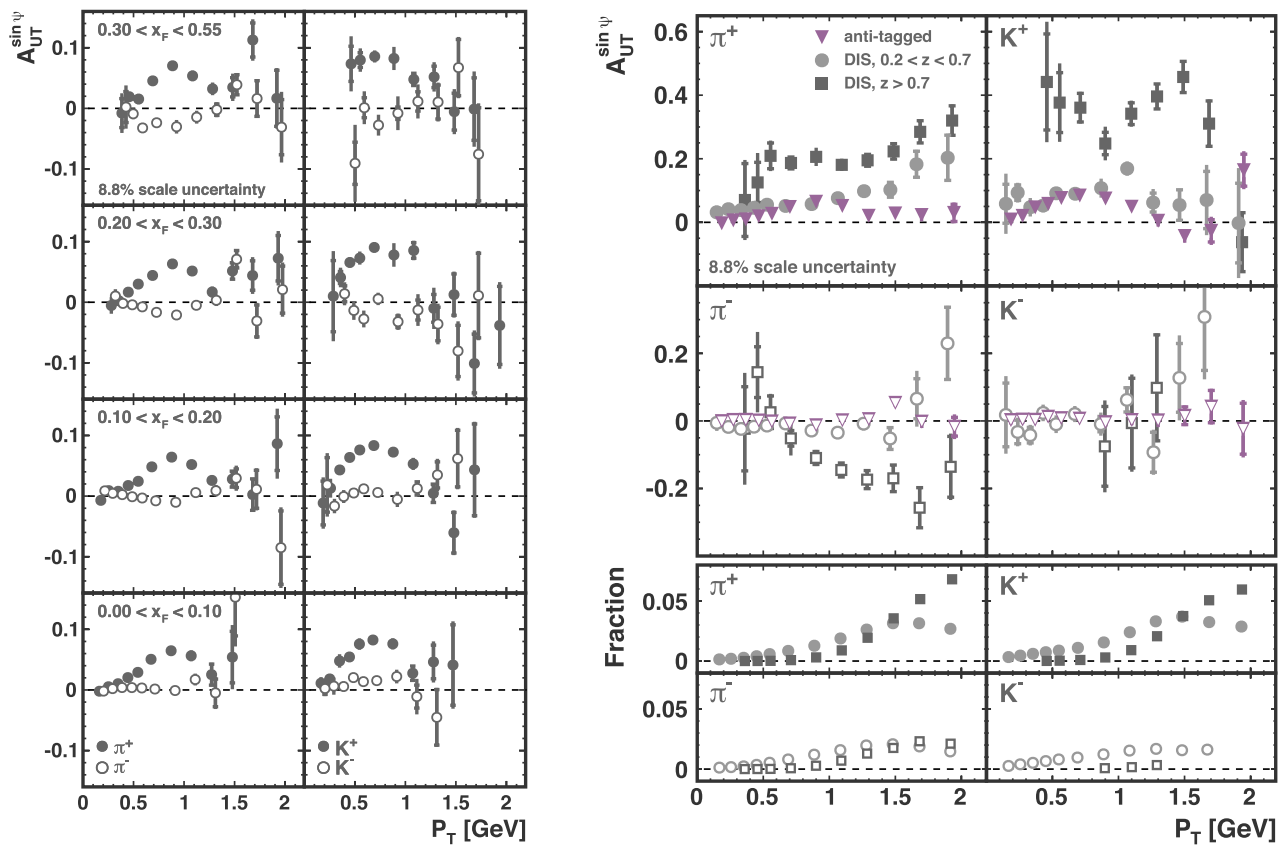

Figure 2: $A_{U T}^{\sin \psi}$ amplitudes for charged pions and kaons as a function of $P_{T}$ for various slices in $x_{F}$ (left) and for the 'anti-tagged' category and the two DIS subsamples with $0.2<\mathrm{z}<0.7$ and $\mathrm{z}>0.7$, respectively (right). The bottom panels of the right figure show the relative fractions of the two DIS subsamples with respect to the total inclusive sample of the corresponding hadron species after correction for trigger efficiency.

More insight, especially concerning the peculiar $P_{T}$ dependence, can be gained by studying separately the asymmetries for events without a scattered lepton in the acceptance ('antitagged' category) and events with a scattered lepton in the acceptance, especially those fulfilling the kinematic requirements for deep-inelastic scattering ('DIS'), i.e., $Q^{2}>1 \mathrm{GeV}^{2}, W^{2}>10 \mathrm{GeV}^{2}$, $0.023<x<0.4$, and $0.1<y<0.95$, where $W^{2}$ is the squared invariant mass of the virtual-photon nucleon system, $x$ is the Bjorken scaling variable and $y$ is the fractional beam energy carried by the virtual photon in the laboratory system. After correction for trigger efficiency, about $98 \%$ of all hadrons belong to the 'anti-tagged' category. The fraction of these hadrons with respect to the total inclusive sample is nearly $100 \%$ at low $P_{T}$. It decreases monotonically to about $85-90 \%$ for positive hadrons and to more than $90 \%$ for negative hadrons at the highest $P_{T}$ values. For this category, the undetected lepton in most cases had a small scattering angle and remained within the beam pipe. This data sample is dominated by the kinematic regime $Q^{2} \approx 0 \mathrm{GeV}^{2}$ of quasi-real photoproduction. Hence, $P_{T}$ is the only hard scale, while for the DIS-events $\left\langle Q^{2}\right\rangle$ is always larger than $\left\langle P_{T}^{2}\right\rangle$. The 'DIS' sample has further been divided into two subsamples with $0.2<z<0.7$ and $z>0.7$, where $z$ is the fractional virtual-photon energy carried by the hadron in the laboratory system. The former subsample is identical to the one used previously $[16,17]$ for the determination of azimuthal transverse single-spin asymmetries in semi-inclusive deep-inelastic scattering related to the Sivers and transversity distributions and the Collins fragmentation function.

In the right panel of Figure 2, the $A_{U T}^{\sin \psi}$ amplitudes are presented as a function of $P_{T}$ for the 
'anti-tagged' category and the two DIS subsamples. Also shown are the relative fractions of these two subsamples with respect to the total inclusive sample of the corresponding hadron species after correction for trigger efficiency. At high $P_{T}$, the 'anti-tagged' asymmetry amplitude is consistently smaller than the inclusive amplitude for positive pions and its $P_{T}$ dependence is, within uncertainties, compatible with a constant or a decrease with $P_{T}$. The origin of the observed asymmetry can most likely be explained by higher-twist contributions. At $P_{T}>1.3 \mathrm{GeV}$ the contributions from the other subsamples become sizeable causing the increase with $P_{T}$ observed for the inclusive asymmetry amplitude. For the DIS events with $0.2<z<0.7$ the observed asymmetries are very similar to those in Ref. [16] and we can conclude that the $P_{T}$ dependence is dominantly caused by the Sivers effect as predicted in Ref. [18]. The asymmetries for the DIS events with $z>0.7$ are even larger, reaching values of over $0.4(0.3)$ for $K^{+}\left(\pi^{+}\right)$and -0.25 for $\pi^{-}$. In this kinematic regime of high $z$, exclusive processes can contribute substantially to the asymmetry. Especially remarkable is the large negative amplitude observed for negative pions. This may indicate that a large fraction of events in this subsample stems from the favoured fragmentation of the struck quark (here the down-quark) and that the asymmetry possibly preserves information from the down-quark Sivers function without dilution from disfavoured fragmentation of the otherwise dominating up-quark.

\section{References}

[1] U. D’Alesio and F. Murgia, Prog. Part. Nucl. Sci. 61 (2008) 394 [0712.4328].

[2] V. Barone, F. Bradamante and A. Martin, Prog. Part. Nucl. Sci. 65 (2010) 267 [1011.0909].

[3] J. C. Collins, Nucl. Phys. B 396 (1993) 161 [hep-ph/9208213].

[4] D. W. Sivers, Phys. Rev. D 41 (1990) 83.

[5] J. Qiu and G. F. Sterman, Phys. Rev. D 59 (1999) 014004 [hep-ph/9806356].

[6] Y. Koike, Nucl. Phys. A 721 (2003) 364 [hep-ph/0211400].

[7] C. Kouvaris, J. Qiu, W. Vogelsang and F. Yuan, Phys. Rev. D 74 (2006) 114013 [hep-ph/0609238].

[8] Y. Koike, W. Vogelsang and F. Yuan, Phys. Lett. B 659 (2008) 878 [0711.0636].

[9] Z.-B. Kang et al., Phys. Rev. D 83 (2011) 094001 [1103.1591].

[10] M. Anselmino et al., J. Phys. Conf. Ser. 295 (2011) 012062 [1012.3565].

[11] M. Anselmino et al., Phys. Rev. D 87 (2013) 094019 [1303.3822].

[12] A. Airapetian et al. (HERMES Collab.), Phys. Lett. B 728 (2014) 183 [1310.5070].

[13] K. Ackerstaff et al. (HERMES Collab.), Nucl. Instr. Meth. A 417 (1998) 230 [hep-ex/9806008].

[14] A. Airapetian et al. (HERMES Collab.), Nucl. Instr. Meth. A 540 (2005) 68 [physics/0408137].

[15] C. Aidala, in proceedings of the Second Workshop on Transverse Polarization Phenomena in Hard Processes, World Scientific, Singapore 2009, 112.

[16] A. Airapetian et al. (HERMES Collab.), Phys. Rev. Lett. 103 (2009) 152002 [0906.3918].

[17] A. Airapetian et al. (HERMES Collab.), Phys. Lett. B 693 (2010) 11 [1006.4221].

[18] M. Anselmino et al., Phys. Rev. D 81 (2010) 034007 [0911.1744]; [1404.6465]. 